\title{
A Synchronous Transmission Method for Array Signals of Sensor Network under Resonance Technology
}

\author{
Huadong Wang \\ School of Computer Science and Technology, Zhoukou Normal University, Zhoukou 466001, China
}

Corresponding Author Email: wanghuadong@zknu.edu.cn

https://doi.org/10.18280/ts.370405

Received: 18 April 2020

Accepted: 29 June 2020

\section{Keywords:}

resonance technology, wavelet transform, sensor network, array signals, three-node collaboration

\begin{abstract}
The traditional transmission methods for array signals face problems like signal loss and inaccurate output, due to the inadequacy of signal processing. To solve the problems, this paper presents a synchronous transmission method for array signals of sensor network under resonance technology. For better transmission efficiency, the array signals were collected through three-node collaboration in the sensor network, and denoised through wavelet transform. After that, the abnormal nodes in the sensor network were detected to improve transmission accuracy. On this basis, vibration frequency of the array signals was adjusted by the degree of harmonic vibration. Finally, the synchronous and accurate transmission of array signals was realized through normalization and adaptive solution of echo signals. Experimental results show that the proposed method achieved greater information throughput and higher transmission accuracy than traditional methods within the same time. Therefore, this research provides a highly applicable synchronous transmission method for array signals.
\end{abstract}

\section{INTRODUCTION}

Sensor technology is widely recognized for its excellence in automatic detection and measurement. If multiple sensors with communication and computing capabilities are deployed in the same area, they will self-organize into a distributed intelligent network called sensor network. In the sensor network, the signals from several sensors, which are arranged by a certain rule, will form an array of signals commonly referred to as the array signals. The accurate transmission of the array signals directly bears on the accuracy and reliability of the output of the sensor network.

Jagatheswari et al. [1] proposed a carrier-based synchronous transmission method for wireless signals. First, system modeling was performed by the coupling theory to analyze the features and efficiency of signal synchronous transmission. Then, the adjustable voltage regulator circuit was used to modulate the transmitter voltage. After modulation, the pickup end of the system collects energy through the coupling mechanism, and restores the signals through carrier analysis. On the upside, their method can realize energy-carrying communication without changing the topology of the transmission system. On the downside, the information throughput is small and signal losses are frequent, due to the mismatch between the coupling mechanism and the pickup end. Guner et al. [2] designed a synchronous transmission method for energy and signals with a parallel inductive structure of fundamental energy channel and harmonic energy channel. By this method, the power distribution between the two channels is adjusted to transmit the energy and signals without changing the system resonance. The effectiveness of the method is independent of the output voltage and load of the system. But the accuracy of synchronous transmission needs to be further improved.
Considering the features of array signals, this paper presents a synchronous transmission method for array signals of sensor network under resonance technology. The array signals were collected through three-node collaboration: three nodes were evenly placed by an equal interval, and the arrival time of the target recorded by the central node was taken as the standard time. Based on the time differences and distances between the three nodes, the mean velocity of the target passing through the three-node region was calculated. To detect the array signals more accurately, the wavelet analysis was performed to denoise the collected signals, and to identify the abnormal nodes in time. Finally, the vibration frequency of the array signals was adjusted by resonance technology, completing the synchronous transmission of the array signals. The proposed method was proved highly feasible through experiments.

\section{METHODOLOGY}

\subsection{Array signal collection through three-node collaboration}

Following the principle of three-node collaborative target measurement in the sensor network, three nodes were evenly placed by an equal interval, and the arrival time of the target recorded by the central node was taken as the standard time. Then, the mean velocity of the target passing through the three-node region was derived from the time differences and distances between the three nodes.

Let $L$ be the interval between the three nodes. When the target passes through node $S_{l}$, this node will send a signal to $S_{2}$. The latter node will record the arrival time $t_{1}$ of the target at node $S_{1}$. When the target passes through node $S_{2}$, this node will record the arrival time $t_{2}$ of the target at it. The arrival time 
$t_{3}$ of the target at node $S_{3}$ is recorded by node $S_{2}$ in a similar manner. Then, the velocity $v$ of the target transmission can be expressed as:

$$
V=\frac{1}{3}\left(\frac{L}{t_{2}-t_{1}}+\frac{L}{t_{3}-t_{2}}+\frac{L}{t_{3}-t_{1}}\right)
$$

There are three advantages of three-node collaboration in the detection of target velocity:

(1) Eliminate the complicated identification of target type;

(2) Avoid the hardware clock difference between nodes;

(3) Realistically reflect the target velocity in the data collection region with the mean velocity [3-7].

It is assumed that any four nodes in the sensor network can form a rectangle, and the minimize interval between two nodes is $40 \mathrm{~mm}$. Then, the minimum network was constructed with three nodes. When a target enters the data collection region, a model can be established as shown in Figure 1.

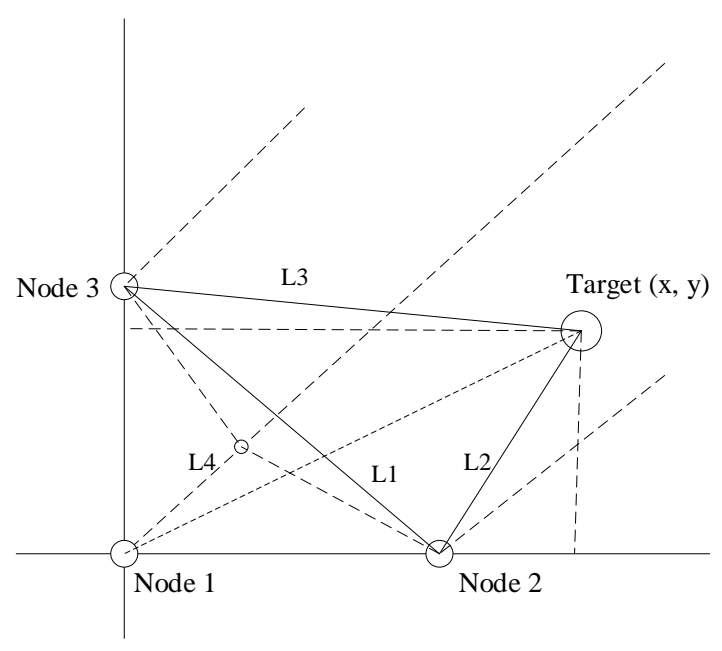

Figure 1. Three-node positioning

The moving direction and position of the target can be determined by computing the coordinates of the target in the above relative coordinate system. Based on geometric relations, the following can be derived from Figure 1 .

By the law of cosines [8-10]:

$$
\begin{aligned}
& L_{3}^{2}=L_{1}^{2}+L_{2}^{2}-2 L_{1} L_{2} \cos C_{2} \\
& L_{2}^{2}=L_{1}^{2}+L_{3}^{2}-2 L_{1} L_{3} \cos C_{3}
\end{aligned}
$$

Formulas (2) and (3) can be transformed into:

$$
\begin{aligned}
& \cos C_{2}=\frac{L_{1}^{2}+L_{2}^{2}-L_{3}^{2}}{2 L_{1} L_{2}} \\
& \cos C_{3}=\frac{L_{1}^{2}+L_{3}^{2}-L_{2}^{2}}{2 L_{1} L_{3}}
\end{aligned}
$$

Thus:

$$
C_{2}=\arccos \left(\frac{L_{1}^{2}+L_{2}^{2}-L_{3}^{2}}{2 L_{1} L_{2}}\right)
$$

$$
C_{3}=\arccos \left(\frac{L_{1}^{2}+L_{3}^{2}-L_{2}^{2}}{2 L_{1} L_{3}}\right)
$$

From the geometric relations in Figure 1:

$$
\begin{gathered}
x=L_{3} \sin \left(C_{3}+45^{\circ}\right) \\
y=L_{2} \sin \left(135^{\circ}-C_{2}\right)
\end{gathered}
$$

Substituting formulas (6) and (7) into formulas (8) and (9):

$$
\begin{aligned}
& x=L_{3} \sin \left[\arccos \left(\frac{L_{1}^{2}+L_{3}^{2}-L_{2}^{2}}{2 L_{1} L_{3}}\right)+45^{\circ}\right] \\
& y=L_{2} \sin \left[135^{\circ}-\arccos \left(\frac{L_{1}^{2}+L_{2}^{2}-L_{3}^{2}}{2 L_{1} L_{2}}\right)\right]
\end{aligned}
$$

The azimuth angle $\theta=\arctan \frac{y}{x}$ can be expressed as:

$$
\theta=\arctan \frac{y}{x} \frac{L_{2} \sin \left(135^{\circ}-\arccos \frac{L_{1}^{2}+L_{2}^{2}-L_{3}^{2}}{2 L_{1} L_{2}}\right)}{L_{3} \sin \left(\arccos \frac{L_{1}^{2}+L_{3}^{2}-L_{2}^{2}}{2 L_{1} L_{3}}+45^{\circ}\right)}
$$

Through the above process, the moving direction and coordinates of the target can be obtained. For the established minimum sensor network, $C=35^{\circ}, L=40 \mathrm{~m}$, and $L_{1}=\sqrt{2 L}$. The coordinates of the target can be calculated accurately, once the values of $L_{2}$ and $L_{3}$ are determined.

The above calculation process shows that the array signals will attenuate with the growing transmission distance. The following can be determined by analyzing the traditional experimental data: the array signal amplitude [11-15], and the distance relationships between target and nodes. Therefore, the distances of the array signals $L_{2}$ and $L_{3}$ in the nodes can be derived from their amplitude.

In this way, the $x$ and $y$ values can be calculated accurately. Then, the moving direction and position of the target can be determined in the coordinate system of Figure 1: the coordinates are $(x, y)$ and the azimuth angle is $\theta=\arctan (y / x)$. This three-node collaboration method is very easy to implement. Besides, the key parameters $L_{2}$ and $L_{3}$ for solving the target coordinates can be pinpointed through three-node collaboration, making it possible to position and collect array signals.

\subsection{Array signal denoising through wavelet analysis}

The array signals collected from the sensor network contain lots of noises. Some of these noisy signals will be lost during transmission [16-18]. To prevent signal loss, wavelet analysis was performed to filter out the noises.

The collected array signals $\Psi(t) \in L^{2}(R)$ are a basic wavelet or mother wavelet, if its Fourier transform result $\Psi(\omega)$ satisfies:

$$
\Psi(\omega)=\int_{R} \frac{|\Psi(t)|^{2}}{|\omega|} d \omega
$$

where, $\omega$ is frequency. 
By translating and scaling the mother wavelet $\Psi(t)$, it is possible to derive a wavelet sequence [19]. If it is continuous, the wavelet sequence can be expressed as:

$$
\Psi_{a, b}(t)=\frac{1}{\sqrt{|a|}} \Psi\left(\frac{t-b}{a}\right)
$$

where, $a(a \neq 0)$ is the scaling factor; $b$ is the translation factor.

For a random function $f(t) \in L^{2}(R)$, the continuous wavelet transform can be expressed as:

$$
W_{f}(a, b)=\left\langle f, \Psi_{a, b}\right\rangle|a|^{-1 / 2} \int_{R} f(t) \Psi\left(\frac{t-b}{a}\right) d t
$$

Under binary discrete state, the wavelet sequence can be expressed as:

$$
\Psi_{j, k}(a, b)=2^{-j / 2} \Psi\left(2^{-j}, t-k\right) j, k \in z
$$

The one-dimensional (1D) noisy signals can be described as:

$$
y_{i}=f_{i}+e_{i}
$$

where, $y_{i}$ is the noisy array signals; $f_{i}$ is the real signals; $e_{i}$ is the Gaussian white noise $N\left(0, \sigma^{2}\right)$.

In a real sensor network, useful array signals are usually low-frequency signals, while noise signals are usually highfrequency signals. Therefore, the denoising process can be divided into three steps:

Step 1: Wavelet decomposition of array signals

Select a wavelet and determine the number of decomposed layers $\mathrm{N}$, and then perform wavelet decomposition of the array signals $\mathrm{s}$ into $\mathrm{N}$ layers. Figure 2 explains the three-layer wavelet decomposition. The noises generally exist in highfrequency parts D1-D3, while the useful signals in lowfrequency parts A1-3.

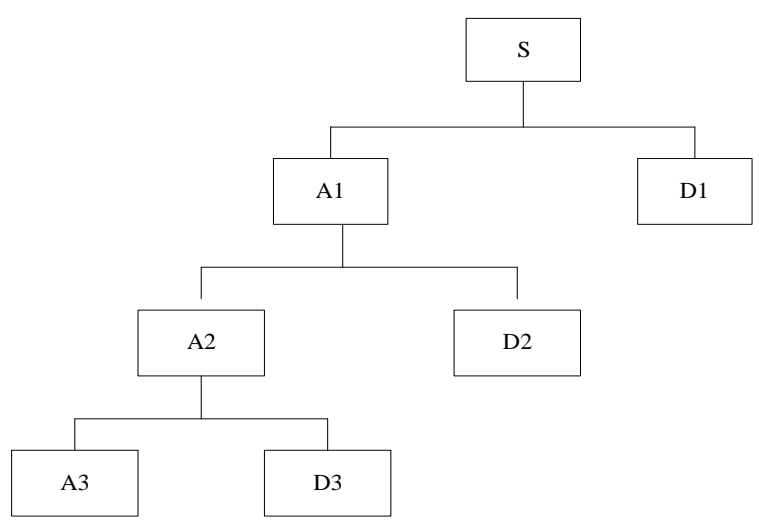

Figure 2. The process of three-layer wavelet decomposition

Step 2: Threshold quantization of high-frequency coefficients

Select a threshold to quantize the high-frequency coefficients from layer 1 to layer $\mathrm{N}$ [20].

Step 3: Wavelet reconstruction

Reconstruct the useful signals with the wavelet of the 1D signals, according to the low-frequency coefficients on layer $\mathrm{N}$ and the quantized high-frequency coefficients of layers 1-N.
The denoising process aims to suppress the noises in the signals, and reveal the real signals.

\subsection{Detection of abnormal nodes}

To further improve the transmission accuracy, it is necessary to detect the abnormal nodes in the sensor network. Suppose $f(t) \in L^{2}(R)$, and $\Psi(t)$ is a continuously differentiable wavelet with an n-order vanishing moment, satisfying the condition (13). Then, there exists:

$$
|W f(s, t)| \leq K s^{a}
$$

where, $K$ is a constant. Thus, $a$ represents the singularity index of point $t_{0}$, i.e. the Lipschitz exponent.

In fact, if the wavelet basis function $\Psi(t)$ is the first-order derivative of the smoothing function $\theta(t)(\Psi(t)=d \theta(t) / d t)$, then the wavelet transform of function $f(t)$ can be expressed as:

$$
W f(s, t)=f \times \Psi_{s}(t)=f \times\left(s \frac{d \theta_{s}}{d t}\right)(t)
$$

It can be seen that $W f(s, t)$ is proportional to the derivative function of $f(t)$ after being smoothened by $\theta(t)$. On a specific scale $\mathrm{s}$, the maximum of $W f(s, t)$ along the time axis $\mathrm{t}$ corresponds to the mutation point of $f \times \Psi_{s}(t)$. Since $\theta(t)$ is differentiable, the coordinates of the extreme value of $W f(s, t)$ should appear near the modulus maximum point of $f(t)$, if $\theta_{s}(t)$ has a sufficiently small equivalent width. In other words, the mutation point of the original signals can be identified by searching for the modulus maximum point of the wavelet transform [21-24].

On the binary scale, taking the logarithm on both sides of formula (16):

$$
\log _{2}\left|W f\left(2^{j}, t\right)\right| \leq \log _{2} K+\alpha^{j}
$$

Through the above analysis, since the Lipschitz exponent of the array signals is greater than zero, the modulus maximum value of the wavelet transform of the signals will increase with the scale; since the Lipschitz exponent of white noise is negative [25-27], the modulus maximum value of the wavelet transform of the noise will decrease with the growing scale. Therefore, whether the modulus maximum value arises from noise or abnormal value can be judged by observing its variation between different binary scales $2^{j}$. In this way, the signals can be differentiated from the noises, achieving the denoising effect.

\subsection{Synchronous transmission of array signals under resonance technology}

Before synchronous transmission, the maximum transmission cycle should be adopted when the array signal transmission changes stably, i.e. the array signals vary very slightly, and the minimum cycle should be adopted when the array signal transmission changes significantly, aiming to enhance the ability to capture array signals with significant variations.

Based on the harmonic vibration and the change of the transmission method, this paper puts forward an adaptive synchronization transmission method, which firstly linearly 
fits each sampling point with a unary linear regression equation [28], and then adjusts the vibration frequency of the array signals by resonance technology.

Let $y_{s}=f(x)$ be the regression curve of the sampled array signals. Then, the sampling set $\mathrm{T}$ can be described as:

$$
T=\left\{\left(x_{1}, y_{1}\right),\left(x_{2}, x_{2}\right), \cdots\left(x_{n}, x_{n}\right)\right\}
$$

The sampling points are sampled at times $t_{1}, t_{2}, \cdots, t_{n}$, respectively. The correlations between the sampling times can be expressed as: $t_{1}<t_{2}<\cdots<t_{n}$.

Linking up all nodes in set $T$, a new sampling curve $y^{\prime}$ can be obtained:

$$
y_{s}^{\prime}=f^{\prime}(x)
$$

Suppose the region between curves $y_{s}$ and $y_{s}$ ' represents the distortion of the sampled array signals. Then, $\xi(x)$ can be described as:

$$
\xi(x)=\int\left|f^{\prime}(x)-f(x)\right| d x
$$

On this basis, the latest $M$ sampling points were fitted into a regression curve. Let $y_{i+1}$ be the value of the first sampling point; $t_{i}, t_{i-1}, \ldots t_{i-M}$ be the sampling times of the $M$ points, respectively; $y_{i}, y_{i-1}, \cdots y_{i-M}$ be the values of the $M$ points, respectively. Then, the sampling times of these points satisfy $t_{i}>t_{i-1}>\cdots>t_{i-M}$. The unary linear regression equation for the signals passing through the $M$ points can be defined as:

$$
y_{s}^{\prime}=\varsigma t+\tau
$$

Using the unary linear regression equation, the slope coefficient $\varsigma$ and the constant $\tau$ can be obtained through maximum likelihood estimation [29]:

$$
\left\{\begin{array}{l}
\varsigma=\frac{\sum_{k=i-1}^{i-n}\left(t_{k}-\bar{t}\right)\left(y_{k}-\bar{y}\right)}{\sum_{k=i-1}^{i-n}\left(t_{k}-\bar{t}\right)^{2}} \\
\tau=\bar{y}-\tau \bar{t}
\end{array}\right.
$$

Then, the next sampling time can be derived from the slope coefficient $\varsigma$ of the unary linear regression equation. If $|\varsigma|$ is relatively large, then the sampling time interval will be small; the inverse is also true. During the control of the sampling points, the harmonic signals of array signal discretization should be properly described to improve the transmission accuracy of the array signals. The harmonic signal sequence obtained from the sampled array signals can be expressed as:

$$
\left\{y_{k}: k=1,2, \cdots, n\right\}
$$

Then, numerous harmonic signals, which obey Gaussian distribution, were superimposed, and normalized into:

$$
y=f(t)=\sum_{i=1}^{N-1} \lambda_{i} \exp \left(\frac{-k\left(t_{i}-t\right)^{2}}{t_{2}}\right)
$$

Solving formula (27) by the minimum mean square error (MMSE) method:

$$
Z=\sqrt{\frac{1}{N} \sum_{i=1}^{N-1}\left[f\left(t_{k}-y_{k}\right)\right]^{2}} \geq v
$$

where, $v$ is the required accuracy of signal transmission. Solving formula (28), the echo signal of each sampling point can be obtained, thereby improving the sampling accuracy of the array signals.

Through the above process, the array signals of the sensor network can be transmitted synchronously and accurately under the resonance technology.

\section{EXPERIMENTS AND RESULT ANALYSIS}

To verify the applicability of the proposed synchronous transmission method for array signals, the throughput and accuracy of the synchronous transmission were analyzed through contrastive experiments [30]. The proposed method was compared with the carrier-based synchronous transmission method for wireless signals (Method 1) [1], and the synchronous transmission method for energy and signals with a parallel inductive structure of fundamental energy channel and harmonic energy channel (Method 2) [2].

Figure 3 compares the throughputs of the three methods in synchronous transmission.

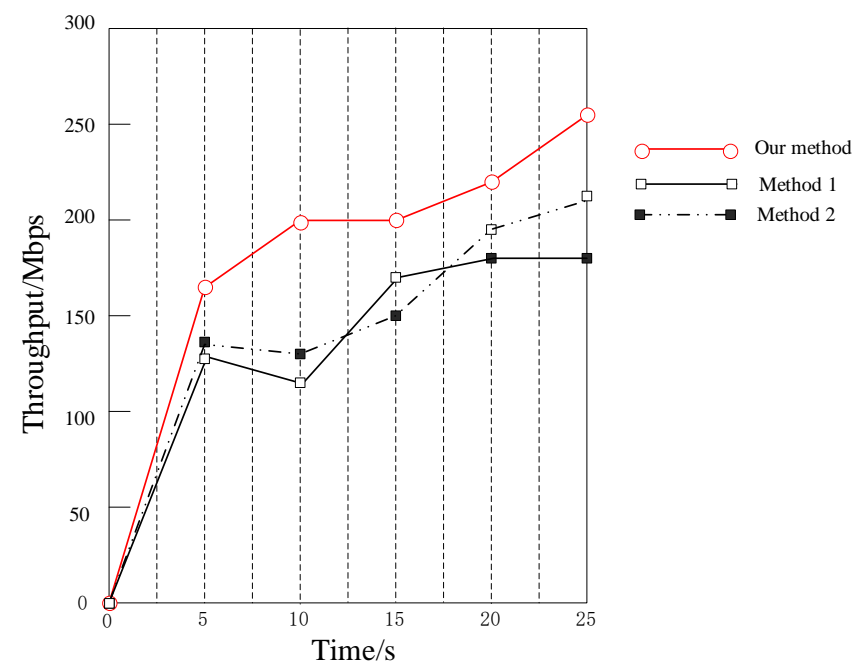

Figure 3. The throughputs of the three methods

As shown in Figure 3, the throughputs of Method 1 and Method 2 both decreased first and then increased, while the throughput of our method remained above $150 \mathrm{MPa}$ and that of the two contrastive method. The comparison shows that our method achieved the greater throughput in the same period of time.

The superiority of our method comes from the denoising operation in our method, which removes the redundant information, making the useful signals pass through the sensor network more effectively.

Table 1 compares the transmission accuracies of the three methods with the growing number of transmission signals.

As shown in Table 1, the transmission accuracies of all three methods declined, as more and more signals were being 
transmitted. However, our method had the smallest decrement in transmission accuracy, and kept the transmission accuracy above $95 \%$. This is because our method timely detects the abnormal nodes in the sensor network, reducing the probability of transmitting incorrect signals. This fully demonstrates the effectiveness of our method.

Table 1. The transmission accuracies of the three methods

\begin{tabular}{cccc}
\hline $\begin{array}{c}\text { Number of } \\
\text { signals/Mbit }\end{array}$ & \multicolumn{3}{c}{ Transmission accuracy /\% } \\
Our method & Method 1 & Method 2 \\
\hline $1 \times 10^{5}$ & 98.73 & 94.14 & 92.26 \\
$1 \times 10^{6}$ & 97.55 & 93.67 & 90.98 \\
$1 \times 10^{7}$ & 97.16 & 90.87 & 89.14 \\
$1 \times 10^{8}$ & 96.34 & 89.21 & 83.24 \\
$1 \times 10^{9}$ & 95.77 & 86.41 & 80.97 \\
\hline
\end{tabular}

\section{CONCLUSIONS}

The array signals of sensor network carry a huge amount of information, and requires a high accuracy in transmission. Therefore, this paper designs a synchronous transmission method for the array signals under the resonance technology, and experimentally proves that the proposed method can transmit the array signals accurately at a higher efficiency. The future research will further optimize the proposed method from the perspective of energy conservation.

\section{ACKNOWLEDGMENT}

This work was supported by Science and Technology Breakthrough Project of Henan Provincial Science and Technology Department under the program "Brain Tumor Image Segmentation Based on Depth Learning" (Grant No.: 182102310694) and "Energy Consumption Modeling and Measurement in Virtual Environment" (Grant No.: 182102310034), and Development Plan Project of Henan Provincial Science and Technology Department under the program "Research and Development of University Financial Intelligent Platform in the Context of Cloud Computing" (Grant No.: 192400410368).

\section{REFERENCES}

[1] Jagatheswari, G.K., Honnavar, G.V., Murugesan, R. (2017). Analysis of some two dimensional functions using two dimensional Fourier transforms: Image reconstruction and physical significance. In 2017 2nd International Conference on Computing and Communications Technologies (ICCCT), pp. 310-313. https://doi.org/10.1109/ICCCT2.2017.7972295

[2] Guner, K.K., Erkmen, B., Gulum, T.O., Erdogan, A.Y., Yildırım, T., Ata, L.D. (2016). Implementation aspects of Wigner-Hough Transform based detectors for LFMCW signals. In 2016 39th International Conference on Telecommunications and Signal Processing (TSP), pp. 441-444. https://doi.org/10.1109/TSP.2016.7760916

[3] Candan, C., Kutay, M.A., Ozaktas, H.M. (2000). The discrete fractional Fourier transform. IEEE Transactions on Signal Processing, 48(5): 1329-1337. https://doi.org/10.1109/78.839980

[4] Biondi, F. (2017). Low-rank plus sparse decomposition and localized radon transform for ship-wake detection in synthetic aperture radar images. IEEE Geoscience and Remote Sensing Letters, 15(1): 117-121. https://doi.org/10.1109/LGRS.2017.2777264

[5] Biondi, F. (2018). A polarimetric extension of low-rank plus sparse decomposition and radon transform for ship wake detection in synthetic aperture radar images. IEEE Geoscience and Remote Sensing Letters, 16(1): 75-79. https://doi.org/10.1109/LGRS.2018.2868365

[6] Swain, R.R., Khilar, P.M., Dash, T. (2020). Multifault diagnosis in WSN using a hybrid metaheuristic trained neural network. Digital Communications and Networks, 6(1): 86-100. https://doi.org/10.1016/j.dcan.2018.02.001

[7] Koushanfar, F., Potkonjak, M., Sangiovanni-Vincentelli, A. (2003). On-line fault detection of sensor measurements. In SENSORS, 2003 IEEE, pp. 974-979. https://doi.org/10.1109/ICSENS.2003.1279088

[8] Lee, M.H., Choi, Y.H. (2008). Fault detection of wireless sensor networks. Computer Communications, 31(14): 3469-3475.

https://doi.org/10.1016/j.comcom.2008.06.014

[9] Mahapatro, A., Khilar, P.M. (2014). Online fault diagnosis of wireless sensor networks. Central European Journal of Computer Science, 4(1): 30-44. https://doi.org/10.2478/s13537-014-0203-8

[10] Jutten, C., Herault, J. (1991). Blind separation of sources, part I: An adaptive algorithm based on neuromimetic architecture. Signal Processing, 24(1): 1-10. https://doi.org/10.1016/0165-1684(91)90079-X

[11] Aysal, T.C., Barner, K.E. (2008). Blind decentralized estimation for bandwidth constrained wireless sensor networks. IEEE Transactions on Wireless Communications, $\quad 7(5)$ : 1466-1471. https://doi.org/10.1109/TWC.2008.060687

[12] Hallez, H., De Vos, M., Vanrumste, B., Van Hese, P., Assecondi, S., Van Laere, K., Dupont, P., Van Paesschen, W., Van Huffel, S., Lemahieu, I. (2009). Removing muscle and eye artifacts using blind source separation techniques in ictal EEG source imaging. Clinical Neurophysiology, $120(7)$ : $1262-1272$. https://doi.org/10.1016/j.clinph.2009.05.010

[13] Nakajima, H., Nakadai, K., Hasegawa, Y., Tsujino, H. (2009). Blind source separation with parameter-free adaptive step-size method for robot audition. IEEE Transactions on Audio, Speech, and Language Processing, 18(6): 1476-1485. https://doi.org/10.1109/TASL.2009.2035219

[14] Rodriguez, A., Laio, A. (2014). Clustering by fast search and find of density peaks. Science, 344(6191): 14921496. https://doi.org/10.1126/science. 1242072

[15] Hinton, G.E., Osindero, S., Teh, Y.W. (2006). A fast learning algorithm for deep belief nets. Neural Computation, $18(7)$ : https://doi.org/10.1162/neco.2006.18.7.1527

[16] Sangeetha, J., Jayasankar, T. (2019). Emotion speech recognition based on adaptive fractional deep belief network and reinforcement learning. In Cognitive Informatics and Soft Computing, pp. 165-174. https://doi.org/10.1007/978-981-13-0617-4_16

[17] Srivastava, N., Hinton, G., Krizhevsky, A., Sutskever, I., Salakhutdinov, R. (2014). Dropout: A simple way to prevent neural networks from overfitting. The journal of Machine Learning Research, 15(1): 1929-1958. https://doi.org/10.5555/2627435.2670313 
[18] Hinton, G.E. (2012). A practical guide to training restricted Boltzmann machines. In Neural Networks: Tricks of the Trade, pp. 599-619. https://doi.org/10.1007/978-3-642-35289-8_32

[19] Sheinker, A., Salomonski, N., Ginzburg, B., Frumkis, L., Kaplan, B.Z. (2008). Magnetic anomaly detection using entropy filter. Measurement Science and Technology, 19(4): $\quad 045205 . \quad$ https://doi.org/10.1088/09570233/19/4/045205

[20] Kakarala, R., Ogunbona, P.O. (2001). Signal analysis using a multiresolution form of the singular value decomposition. IEEE Transactions on Image Processing, 10(5): 724-735. https://doi.org/10.1109/83.918566

[21] Benzi, R., Sutera, A., Vulpiani, A. (1981). The mechanism of stochastic resonance. Journal of Physics A: Mathematical and General, 14(11): L453. https://doi.org/10.1088/0305-4470/14/11/006

[22] Wang, C., Huang, N., Bai, Y., Zhang, S. (2018). A method of network topology optimization design considering application process characteristic. Modern Physics Letters B, 32(7): 1850091. https://doi.org/10.1142/S0217984918500914

[23] Diamant, R., Francescon, R., Zorzi, M. (2017). Topology-efficient discovery: A topology discovery algorithm for underwater acoustic networks. IEEE Journal of Oceanic Engineering, 43(4): 1200-1214. https://doi.org/10.1109/JOE.2017.2716238

[24] Cavraro, G., Arghandeh, R. (2017). Power distribution network topology detection with time-series signature verification method. IEEE Transactions on Power Systems, 33(4): 3500-3509. https://doi.org/10.1109/TPWRS.2017.2779129
[25] Lee, K., Wu, Y., Bresler, Y. (2017). Near-optimal compressed sensing of a class of sparse low-rank matrices via sparse power factorization. IEEE Transactions on Information Theory, 64(3): 1666-1698. https://doi.org/10.1109/TIT.2017.2784479

[26] Parsegov, S.E., Proskurnikov, A.V., Tempo, R., Friedkin, N.E. (2016). Novel multidimensional models of opinion dynamics in social networks. IEEE Transactions on Automatic Control, 62(5): 2270-2285. https://doi.org/10.1109/TAC.2016.2613905

[27] Corsini, G., Mossa, A., Verrazzani, L. (1996). Signal-tonoise ratio and autocorrelation function of the image intensity in coherent systems. Sub-Rayleigh and superRayleigh conditions. IEEE Transactions on Image Processing, 5(1): https://doi.org/10.1109/83.481677

[28] Yatsenko, V., Kolesnik, Y., Titarenko, T. (1994). Identification of the non-Gaussian chaotic dynamics of the radioemission back scattering processes. IFAC Proceedings Volumes, 27(8): 277-281. https://doi.org/10.1016/S1474-6670(17)47728-7

[29] Mukherjee, S., Osuna, E., Girosi, F. (1997). Nonlinear prediction of chaotic time series using support vector machines. In Neural Networks for Signal Processing VII. Proceedings of the 1997 IEEE Signal Processing Society Workshop, pp. 511-520. https://doi.org/10.1109/NNSP.1997.622433

[30] Leung, H., Hennessey, G., Drosopoulos, A. (2000). Signal detection using the radial basis function coupled map lattice. IEEE Transactions on Neural Networks, 11(5): 1133-1151. https://doi.org/10.1109/72.870045 